\title{
STABILITY OF JUNGCK-TYPE ITERATIVE PROCEDURES
}

\author{
S. L. SINGH, CHARU BHATNAGAR, AND S. N. MISHRA \\ Received 2 December 2004 and in revised form 9 September 2005
}

We introduce and discuss the stability of Jungck and Jungck-Mann iterative procedures for a pair of Jungck-Osilike-type maps on an arbitrary set with values in a metric or linear metric space.

\section{Introduction}

Let $(X, d)$ be a metric space and $T: X \rightarrow X$. Several physical problems, expressed as a fixed point equation $T x=x$, are solved by approximating a sequence $\left\{x_{n}\right\} \subset X$ generated by an iterative procedure $f\left(T, x_{n}\right)$. Let the sequence $\left\{x_{n}\right\}$ converge to a fixed point of $T$. The iterative procedure $f\left(T, x_{n}\right)$ is considered numerically stable if and only if a sequence $\left\{y_{n}\right\} \subset X$ approximatively close to $\left\{x_{n}\right\}$ converges to the desired solution of the equation $T x=x$. This kind of study in $R^{1}$ was initiated by Urabe [32] during the middle of the last century (see also, Collatz [3] and Ortega and Rheinboldt [17]). However, a formal definition of the stability of general iterative procedures is due to Harder and Hicks [7, 8] (cf. definition below). Ostrowski [20] appears to be the first to discuss the stability of iterative procedures on metric spaces. Due to its increasing importance in computational mathematics, especially due to the revolution in computer programming, the stability theory has extensively been studied by various authors (see, e.g., Berinde [1], Czerwik et al. [4], Istrăţescu [9], Jachymski [11], Matkowski and Singh [15], Osilike [18, 19], Rus et al. [27], Rhoades $[24,25]$, and several references of $[1,27])$. However, in all these references cited above, special cases of the following fundamental iterative procedure

$$
x_{n+1}=f\left(T, x_{n}\right), \quad n=0,1, \ldots,
$$

have been studied. As discussed by Harder [6], Harder and Hicks [7, 8], and Berinde [1], the study of stability of iterative procedures is both of theoretical and numerical interest. We introduce the notion of the stability of the iterative procedure ( $\mathrm{S}-\mathrm{HH}$ ) (see below) for a pair of self-maps and develop the theory for this general procedure. Notice that $(\mathrm{S}-\mathrm{HH})$ reduces to $(\mathrm{HH})$ when $Y=X$ and $S=i d$, the identity map on $X$. It may be of interest to note that, besides including the Jungck iterations (see below), (S-HH) includes the well-known Picard, Mann, and a host of other iterative procedures. 


\section{Preliminaries}

Throughout the paper, let $Y$ be an arbitrary nonempty set and $(X, d)$ a metric space. Let $S, T: Y \rightarrow X$ and $T(Y) \subseteq S(Y)$. For any $x_{0} \in Y$, consider

$$
S x_{n+1}=f\left(T, x_{n}\right), \quad n=0,1, \ldots
$$

For $Y=X$ and $f\left(T, x_{n}\right)=T x_{n}$, the iterative procedure (S-HH) yields the Jungck iterations (or J-iterations in brief), namely, $S x_{n+1}=T x_{n}, n=0,1, \ldots$ This procedure was essentially introduced by Jungck [12], and it becomes the Picard iterative procedure when $S=i d$, the identity map on $X$. Jungck [12] showed that the maps $S$ and $T$ satisfying

$$
d(T x, T y) \leq k d(S x, S y), \quad 0 \leq k<1,
$$

for all $x, y \in X$ have a unique common fixed point in complete $X$, provided that $S$ and $T$ are commuting, $T(X) \subseteq S(X)$, and $S$ is continuous. However, the following significantly improved version of this result is generally called the Jungck contraction principle.

Theorem JS [30]. Let $S, T: Y \rightarrow X$ satisfy $(J)$ for all $x, y \in Y$. If $T(Y) \subseteq S(Y)$ and $S(Y)$ or $T(Y)$ is a complete subspace of $X$, then $S$ and $T$ have a coincidence. Indeed, for any $x_{0}$ in $Y$, there exists a sequence $\left\{x_{n}\right\}$ in $Y$ such that

(a) $S x_{n+1}=T x_{n}, n=0,1, \ldots$,

(b) $\left\{S x_{n}\right\}$ converges to $S z$ for some $z$ in $Y$, and $S z=T z$, that is, $S$ and $T$ have a coincidence at $z$.

Further, if $Y=X$ and $S, T$ commute (just) at $z$, then $S$ and $T$ have a unique common fixed point.

A pair of maps $S, T$ satisfying (J) is generally termed as Jungck contraction or J-contraction (cf. Singh [30] and Tivari and Singh [31]). We remark that neither Theorem JS nor any of all the existing generalizations of Jungck's common fixed point theorem [12] says nothing about the convergence of $\left\{x_{n}\right\}$ when $Y$ is a metric space or $Y=X$ (see also $[5,10,13,16,22,27,28,29,30,31]$ and references thereof). Further, if $z_{i} \in Y$ are such that $S z_{i}=T z_{i}, i=1,2, \ldots, n$, then $S z_{j}=T z_{i}, i, j=1,2, \ldots, n$, that is, under the conditions of Theorem JS, $S$ and $T$ may have many coincidence points, but the coincidence values are the same (cf. Singh [29]). For various applications of J-iterations to numerical problems, one may refer to [30] (see also [16]). For practical applications in numerical computations and other situations, characterization of J-contractivity for real-valued functions is of practical importance. So, first we present the following result, wherein $[a, b]$ (resp., ]$a, b\left[\right.$ ) stands for the closed (resp., open) interval and $S^{\prime} x$ for the derivative of $S$ at $x$.

Theorem $S$ [30]. Let $S, T:[a, b] \rightarrow[a, b]$ be differentiable with $S a \neq S b$. Let $T^{\prime} x$ and $S^{\prime} x$ be not both equal to zero for any $x \in] a, b[$. The pair $(S, T)$ is a J-contraction of $[a, b]$ if and only if there exists a positive number $q<1$ such that $\left|T^{\prime} y\right| \leq q\left|S^{\prime} y\right|$ for all $\left.y \in\right] a, b[$.

Theorem JS, the foregoing discussion, and the fundamental work of Harder and Hicks $[7,8]$ motivate the following definition. 
Definition 2.1. Let $S, T: Y \rightarrow X, T(Y) \subseteq S(Y)$, and $z$ a coincidence point of $T$ and $S$, that is, $S z=T z=p$ (say). For any $x_{0} \in Y$, let the sequence $\left\{S x_{n}\right\}$, generated by the iterative procedure (S-HH), converge to $p$. Let $\left\{S y_{n}\right\} \subset X$ be an arbitrary sequence, and set $\varepsilon_{n}=d\left(S y_{n+1}, f\left(T, y_{n}\right)\right), n=0,1,2, \ldots$. Then the iterative procedure $f\left(T, x_{n}\right)$ will be called $(S, T)$-stable if and only if $\lim _{n} \varepsilon_{n}=0$ implies that $\lim _{n} S y_{n}=p$.

This definition reduces to that of the stability of iterative procedure $(\mathrm{HH})$ due to Harder and Hicks $[7,8]$ when $Y=X$ and $S=i d$. Further, as regards the construction of the sequences $\left\{S x_{n}\right\}$ and $\left\{x_{n}\right\}$ under the procedure (S-HH), we may calculate $a_{1}=$ $f\left(T, x_{0}\right)$ and then may proceed to solve the equation $S x_{1}=a_{1}$. We remark that if the map $S$ is not one-one, then we have multiple choices for $x_{1}$ as we have to find an $x_{1} \in S^{-1} a_{1}$. So, one might suspect complications in writing computer programs for solving equations under the general procedure $(\mathrm{S}-\mathrm{HH})$ or, in particular, under the J-iterations (cf. Theorem JS (a)). The suspicion is blown immediately by the fact that, whatever the choice of $x_{1}$ (in $\left.S^{-1} a_{1}\right)$ is, $S x_{1}$ always equals $a_{1}$. For this kind of computer programs intended to solve numerical problems under J-iterations, one may refer to [30]. However, in actual practice, the choice of $x_{1}$ is approximative and $S x_{1}$ is not exactly equal to $a_{1}$. So, in general, instead of getting an exact sequence $\left\{S x_{n}\right\}$, we get an approximative sequence $\left\{S y_{n}\right\}$, and this is the main problem that stability plays a crucial role in actual numerical computations.

For several examples discussing the practical aspect and theoretical importance of the stability when $Y=X$ and $S$ is the identity operator on $X$ in the above definition, one may refer to Berinde [1] and Harder and Hicks [7, 8].

\section{Main results}

First, we present a basic result for the stability of J-iterations.

Theorem 3.1. Let $S$ and $T$ be maps on an arbitrary set $Y$ with values in $X$ such that $T(Y) \subseteq$ $S(Y)$, and $S(Y)$ or $T(Y)$ is a complete subspace of $X$. Let $z$ be a coincidence point of $T$ and $S$, that is, $S z=T z=p$ (say). Let $x_{0} \in Y$ and let the sequence $\left\{S x_{n}\right\}$, generated by $S x_{n+1}=T x_{n}$, $n=0,1, \ldots$, converge to $p$. Let $\left\{S y_{n}\right\} \subset X$ and define $\varepsilon_{n}=d\left(S y_{n+1}, T y_{n}\right), n=0,1, \ldots$ If the pair $(S, T)$ is a J-contraction, that is, $S$ and $T$ satisfy (J) for all $x, y \in Y$, then

(I) $d\left(p, S y_{n+1}\right) \leq d\left(p, S x_{n+1}\right)+k^{n+1} d\left(S x_{0}, S y_{0}\right)+\sum_{i=0}^{n} k^{n-i} \varepsilon_{i}$; further,

(II) $\lim _{n} S y_{n}=p$ if and only if $\lim _{n} \varepsilon_{n}=0$.

Notice that Theorem 3.1 is Theorem 3.4 with $a=k$ and $L=0$. However, we give a blend of the proof of Theorem 3.1.

Proof. By the triangle inequality and the condition $(\mathrm{J})$,

$$
\begin{aligned}
d\left(p, S y_{n+1}\right) & \leq d\left(p, S x_{n+1}\right)+k d\left(S x_{n}, S y_{n}\right)+\varepsilon_{n} \\
& \leq d\left(p, S x_{n+1}\right)+k\left[d\left(T x_{n-1}, T y_{n-1}\right)+d\left(T y_{n-1}, S y_{n}\right)\right]+\varepsilon_{n}
\end{aligned}
$$

Therefore,

$$
d\left(p, S y_{n+1}\right) \leq d\left(p, S x_{n+1}\right)+k^{2} d\left(S x_{n-1}, S y_{n-1}\right)+k \varepsilon_{n-1}+\varepsilon_{n} .
$$

This process, when repeated $n-1$ times, yields (I). 
3038 Stability of Jungck-type iterative procedures

To prove (II), first suppose that $\lim _{n} S y_{n}=p$. Then,

$$
\begin{aligned}
\varepsilon_{n} & =d\left(S y_{n+1}, T y_{n}\right) \\
& \leq d\left(S y_{n+1}, S x_{n+1}\right)+d\left(T x_{n}, T y_{n}\right) \\
& \leq d\left(S y_{n+1}, S x_{n+1}\right)+k d\left(S x_{n}, S y_{n}\right) \longrightarrow 0, \text { as } n \longrightarrow \infty .
\end{aligned}
$$

Now, suppose that $\lim _{n} \varepsilon_{n}=0$.

Let $A$ denote the lower triangular matrix with entries $a_{n i}=k^{n-i}$. Then, $\lim _{n} a_{n i}=0$ for each $i$ and $\sum_{i=0}^{n} a_{n i}=\left(1-k^{n+1}\right) /(1-k) \rightarrow 1 /(1-k)$ as $n \rightarrow \infty$. Therefore, $A$ is multiplicative, that is, for any convergent sequence $\left\{s_{n}\right\}, \lim _{n} A\left(s_{n}\right):=\{1 /(1-k)\} \lim _{n} s_{n}$ (cf. Rhoades [25, page 692]). Since $\lim _{n} \varepsilon_{n}=0, \lim _{n} \sum_{i=0}^{n} k^{n-i} \varepsilon_{i}=0$. Also, $\lim _{n} S x_{n}=p$ implies that $\lim _{n} d\left(p, S x_{n+1}\right)=0$. This completes the proof.

The following classical result is obtained from Theorem 3.1 with $Y=X$ and $S=i d$.

Corollary 3.2 (Ostrowski [20]). Let $(X, d)$ be a complete metric space and $T: X \rightarrow X$. Let $p \in X$ be a fixed point of $T$. Let $x_{0} \in X$ and $x_{n+1}=T x_{n}$ for $n=0,1,2, \ldots$ Suppose that $\left\{y_{n}\right\} \subset X, \varepsilon_{n}=d\left(y_{n+1}, T y_{n}\right)$. If $T$ is the Banach contraction on $X$ with contraction constant $k$, then

$$
d\left(p, y_{n+1}\right) \leq d\left(p, x_{n+1}\right)+k^{n+1} d\left(x_{0}, y_{0}\right)+\sum_{i=0}^{n} k^{n-i} \varepsilon_{i} .
$$

Also, $\lim _{n} y_{n}=p$ if and only if $\lim _{n} \varepsilon_{n}=0$.

We remark that deriving an inspiration from Park and Bae [21] and using Corollary 3.2, one may have an alternative proof of Theorem 3.1. We give a sketch of the same.

Another proof. With the notations of Theorem 3.1, let $a_{n}=S x_{n}$ and $z_{n}=S y_{n}$. Then, $x_{n} \in$ $S^{-1} a_{n}$ and $y_{n} \in S^{-1} z_{n}$, so that we can write $a_{n+1}=T x_{n} \in T\left(S^{-1} a_{n}\right)$. As a matter of fact, for every $u \in S(Y)$, the set $T\left(S^{-1} u\right)$ is a singleton. Indeed, for $a, b \in T\left(S^{-1} u\right)$, there exist $\alpha, \beta \in S^{-1} u$ such that $a=T \alpha, b=T \beta$, and $S \alpha=u=S \beta$. Therefore,

$$
d(a, b)=d(T \alpha, T \beta) \leq k d(S \alpha, S \beta)=0 \Longrightarrow a=b .
$$

Hence, if we define a map $F: S(Y) \rightarrow T(Y) \subseteq S(Y)$ such that $F(u)=T\left(S^{-1} u\right)$, then $F$ is well defined. Further, as above, for $a \in T\left(S^{-1} u\right)$ and $b \in T\left(S^{-1} v\right)$,

$$
d(F u, F v)=d(a, b)=d(T \alpha, T \beta) \leq k d(S \alpha, S \beta)=k d(u, v)
$$

Consequently, $F$ is a Banach contraction. Thus, the procedure $(\mathrm{S}-\mathrm{HH})$ can be rewritten as

$$
a_{n+1}=F a_{n}
$$

which is of the form $(\mathrm{HH})$. Moreover,

$$
\varepsilon_{n}=d\left(S y_{n+1}, T y_{n}\right)=d\left(z_{n+1}, F z_{n}\right) .
$$

All these show that the conclusions of Theorem 3.1 follow from Corollary 3.2. 
We remark that, following the above construction, one may derive the conclusions (a) and (b) of Theorem JS from the Banach's fixed point theorem.

Consider the following conditions for $S, T: Y \rightarrow X, x, y \in Y$, and for some $k \in(0,1)$ : we give a comparison of these conditions;

$$
\begin{aligned}
& d(T x, T y) \leq k \max \left\{d(S x, S y), d(S x, T x), d(S y, T y), \frac{[d(S x, T y)+d(S y, T x)]}{2}\right\}, \\
& d(T x, T y) \leq k \max \left\{d(S x, S y), \frac{[d(S x, T x)+d(S y, T y)]}{2}, d(S x, T y), d(S y, T x)\right\} \\
& d(T x, T y) \leq k \max \{d(S x, S y), d(S x, T x), d(S y, T y), d(S x, T y), d(S y, T x)\}, \\
& d(T x, T y) \leq a d(S x, S y)+L d(S x, T x), \quad a \in(0,1), L \geq 0 .
\end{aligned}
$$

Proposition 3.3. (i) $(J) \Rightarrow(S) \Rightarrow(R D N)$; (ii) $(J) \Rightarrow(R) \Rightarrow(R D N)$; (iii) (S) and (R) are independent; (iv) (R) $\Rightarrow(J O)$; (v) (S) and (JO) are independent; (vi) (RDN) and (JO) are independent; (vii) reverse implications of (i), (ii), and (iv) are not true.

In view of rigorous comparisons of contracting maps presented by Rhoades [23], it is easy to see (i), (ii), (iv), and (vii). The condition (JO) with $Y=X$ and $S=i d$ is discussed by Osilike [18], and the same conclusion makes (vi) evident (see also Berinde [1, pages 137 and 153]). Proof of (v) is akin to that of (vi).

Theorem JS with $(\mathrm{J})$ replaced by $(\mathrm{R})$ is true (cf. Singh [29]). We remark that maps $S$ and $T$ satisfying (JO) need not have a coincidence point. For example, if $Y=X=\{1,2\}$, $d$ is the discrete metric, $T 1=2, T 2=1$, and $S x=x$ for $x \in X$, then (JO) holds for all $x, y \in Y=X$ with $a=L=1 / 2$ (see also Osilike [18] and Berinde [1, page 137]). Further, the condition (S) was first studied by Singh [28], while (RDN), as a generalization of (S), was studied independently by Ranganathan [22] and Das and Naik [5]. The stability of (R) with $Y=X$ and $S=i d$ has been studied by Rhoades [25] (see Corollary 3.6 below). It may be mentioned that ( $\mathrm{RDN})$ with $Y=X$ and $S=i d$ is the most general contraction condition due to Ćirić [2] (see [5]). Osilike's very general stability result [18] is obtained under the condition (JO) with $Y=X$ and $S=i d$ (see Rhoades and Saliga [26, page 204]).

We now present general stability results for maps satisfying (JO).

Theorem 3.4. Let $S$ and $T$ be maps on an arbitrary set $Y$ with values in $X$ such that $T(Y) \subseteq$ $S(Y)$, and $S(Y)$ or $T(Y)$ is a complete subspace of $X$. Let $z$ be a coincidence point of $T$ and $S$, that is, $S z=T z=p$ (say). Let $x_{0} \in Y$ and the sequence $\left\{S x_{n}\right\}$, generated by $S x_{n+1}=T x_{n}$, $n=0,1, \ldots$, converge to $p$. Let $\left\{S y_{n}\right\} \subset X$ and define $\varepsilon_{n}=d\left(S y_{n+1}, T y_{n}\right), n=0,1, \ldots$ If $(S, T)$ satisfies (JO) for all $x, y \in Y$, then

(III) $d\left(p, S y_{n+1}\right) \leq d\left(p, S x_{n+1}\right)+L \sum_{i=0}^{n} a^{n-i} d\left(S x_{i}, T x_{i}\right)+a^{n+1} d\left(S x_{0}, S y_{0}\right)+\sum_{i=0}^{n} a^{n-i} \varepsilon_{i}$; further,

(IV) $\lim _{n} S y_{n}=p$ if and only if $\lim _{n} \varepsilon_{n}=0$. 
3040 Stability of Jungck-type iterative procedures

Proof. It may be completed following the proof of Theorem 3.5.

Notice that Theorem 3.4 reduces to Theorem 3.1 when $L=0$. Also, the general stability result, given by Osilike [18], is derivable from Theorem 3.4 with $Y=X$ and $S=i d$.

In all that follows, $X$ is a normed linear space and $d$ stands for the metric induced by the norm of $X$. Consider the iterative procedure

$$
S x_{n+1}=\left(1-\alpha_{n}\right) S x_{n}+\alpha_{n} f\left(T, x_{n}\right) \text {, }
$$

where $\left\{\alpha_{n}\right\}$ satisfies (i) $\alpha_{0}=1$, (ii) $0 \leq \alpha_{n} \leq 1$ for $n>0$, (iii) $\sum \alpha_{n}=\infty$, and (iv) $\sum_{j=0}^{n} \alpha_{j} \prod_{i=j+1}^{n}\{1-$ $\left.\alpha_{i}+a \alpha_{i}\right\}$ converges.

We will call it Jungck-Mann iterations or simply JM-iterations. Taking $Y=X, S=i d$, and $f\left(T, x_{n}\right)=T x_{n},(* * *)$ reduces to the following:

$$
x_{n+1}=\left(1-\alpha_{n}\right) x_{n}+\alpha_{n} T x_{n}
$$

with $\left\{\alpha_{n}\right\}$ satisfying (i)-(iv). This process was introduced by Mann [14] and has been found very useful in approximating solutions of fixed point equations (see, e.g., [1, 24, $25,26,27])$.

Now, in the next theorem, we prove that the JM-iterative procedure is $(S, T)$-stable for maps $S$ and $T$ satisfying (JO).

Theorem 3.5. Let $S$ and $T$ be maps on an arbitrary set $Y$ with values in $X$ such that $T(Y) \subseteq$ $S(Y)$, and $S(Y)$ or $T(Y)$ is a complete subspace of $X$. Let $z$ be a coincidence point of $T$ and $S$, that is, $S z=T z=p$ (say). Let $x_{0} \in Y$ and the sequence $\left\{S x_{n}\right\}$, generated by $S x_{n+1}=$ $\left(1-\alpha_{n}\right) S x_{n}+\alpha_{n} T x_{n}, n=0,1, \ldots$, with $\left\{\alpha_{n}\right\}$ satisfying $(i)-(i v)$, converge to $p$. Let $\left\{S y_{n}\right\} \subset X$ and define

$$
\varepsilon_{n}=d\left(S y_{n+1},\left(1-\alpha_{n}\right) S y_{n}+\alpha_{n} T y_{n}\right), \quad n=0,1, \ldots
$$

If the pair ( $S, T)$ satisfies (JO) for all $x, y \in Y$, then

(V)

$$
\begin{aligned}
d\left(p, S y_{n+1}\right) \leq & d\left(p, S x_{n+1}\right)+L \sum_{j=0}^{n} \alpha_{j} \prod_{i=j+1}^{n}\left(1-\alpha_{i}+a \alpha_{i}\right) d\left(S x_{i}, T x_{i}\right) \\
& +\prod_{i=0}^{n}\left(1-\alpha_{i}+a \alpha_{i}\right) d\left(S x_{0}, S y_{0}\right)+\sum_{j=0}^{n} \alpha_{j} \prod_{i=j+1}^{n}\left(1-\alpha_{i}+a \alpha_{i}\right) \varepsilon_{j},
\end{aligned}
$$

where the product is 1 when $j=n$. Further,

(VI) $\lim _{n} S y_{n}=p$ if and only if $\lim _{n} \varepsilon_{n}=0$. 
Proof. By the triangle inequality, (***) and (JO),

$$
\begin{aligned}
d\left(p, S y_{n+1}\right) \leq & d\left(p, S x_{n+1}\right)+d\left(\left(1-\alpha_{n}\right) S x_{n}+\alpha_{n} T x_{n},\left(1-\alpha_{n}\right) S y_{n}+\alpha_{n} T y_{n}\right) \\
& +d\left(\left(1-\alpha_{n}\right) S y_{n}+\alpha_{n} T y_{n}, S y_{n+1}\right) \\
\leq & d\left(p, S x_{n+1}\right)+\left(1-\alpha_{n}\right) d\left(S x_{n}, S y_{n}\right)+\alpha_{n} d\left(T x_{n}, T y_{n}\right)+\varepsilon_{n} \\
\leq & d\left(p, S x_{n+1}\right)+\left(1-\alpha_{n}\right) d\left(S x_{n}, S y_{n}\right)+\alpha_{n}\left[a d\left(S x_{n}, S y_{n}\right)+L d\left(S x_{n}, T x_{n}\right)\right]+\varepsilon_{n} \\
\leq & d\left(p, S x_{n+1}\right)+\left(1-\alpha_{n}+a \alpha_{n}\right) d\left(S x_{n}, S y_{n}\right)+\alpha_{n} L d\left(S x_{n}, T x_{n}\right)+\varepsilon_{n} .
\end{aligned}
$$

Also,

$$
\begin{aligned}
d\left(S x_{n}, S y_{n}\right) \leq & d\left(\left(1-\alpha_{n-1}\right) S x_{n-1}+\alpha_{n-1} T x_{n-1},\left(1-\alpha_{n-1}\right) S y_{n-1}+\alpha_{n-1} T y_{n-1}\right) \\
& +d\left(\left(1-\alpha_{n-1}\right) S y_{n-1}+\alpha_{n-1} T y_{n-1}, S y_{n}\right) \\
\leq & \left(1-\alpha_{n-1}\right) d\left(S x_{n-1}, S y_{n-1}\right)+\alpha_{n-1} d\left(T x_{n-1}, T y_{n-1}\right)+\varepsilon_{n-1} \\
\leq & \left(1-\alpha_{n-1}\right) d\left(S x_{n-1}, S y_{n-1}\right) \\
& +\alpha_{n-1}\left[a d\left(S x_{n-1}, S y_{n-1}\right)+L d\left(S x_{n-1}, T x_{n-1}\right)\right]+\varepsilon_{n-1} \\
\leq & \left(1-\alpha_{n-1}+a \alpha_{n-1}\right) d\left(S x_{n-1}, S y_{n-1}\right)+\alpha_{n-1} L d\left(S x_{n-1}, T x_{n-1}\right)+\varepsilon_{n-1} .
\end{aligned}
$$

Therefore,

$$
\begin{aligned}
d\left(p, S y_{n+1}\right) \leq & d\left(p, S x_{n+1}\right)+\left(1-\alpha_{n}+a \alpha_{n}\right)\left(1-\alpha_{n-1}+a \alpha_{n-1}\right) d\left(S x_{n-1}, S y_{n-1}\right) \\
& +\left(1-\alpha_{n}+a \alpha_{n}\right) \alpha_{n-1} L d\left(S x_{n-1}, T x_{n-1}\right)+\alpha_{n} L d\left(S x_{n}, T x_{n}\right) \\
& +\left(1-\alpha_{n}+a \alpha_{n}\right) \varepsilon_{n-1}+\varepsilon_{n} .
\end{aligned}
$$

This process, when repeated $n-1$ times, yields $(\mathrm{V})$.

To prove (VI), suppose that $\lim _{n} S y_{n}=p$. Then,

$$
\begin{aligned}
\varepsilon_{n} & =d\left(S y_{n+1},\left(1-\alpha_{n}\right) S y_{n}+\alpha_{n} T y_{n}\right) \\
& \leq d\left(S y_{n+1}, p\right)+\left(1-\alpha_{n}\right) d\left(p, S y_{n}\right)+\alpha_{n} d\left(p, T y_{n}\right) \\
& \leq d\left(S y_{n+1}, p\right)+\left(1-\alpha_{n}\right) d\left(p, S y_{n}\right)+\alpha_{n} d\left(T p, T y_{n}\right) \\
& \leq d\left(S y_{n+1}, p\right)+\left(1-\alpha_{n}\right) d\left(p, S y_{n}\right)+\alpha_{n}\left[a d\left(S p, S y_{n}\right)+L d(S p, T p)\right] \longrightarrow 0, \quad \text { as } n \longrightarrow \infty .
\end{aligned}
$$

Now, suppose that $\lim _{n} \varepsilon_{n}=0$. Let $A$ denote the lower triangular matrix with entries

$$
b_{n j}=\alpha_{j} \prod_{i=j+1}^{n}\left(1-\alpha_{i}+a \alpha_{i}\right) .
$$

Hence, the condition (iii) implies that this product diverges. Hence, $\lim _{n} b_{n j}=0$ for each $j$. Now, (iv) implies that the limit of the row sum exists. Therefore, $A$ is multiplicative. 
Also, $\lim _{n} S x_{n}=p$ implies that $\lim _{n} d\left(S x_{n}, T x_{n}\right)=0$. Thus,

$$
\lim _{n} L \sum_{j=0}^{n} \alpha_{j} \prod_{i=j+1}^{n}\left(1-\alpha_{i}+a \alpha_{i}\right) d\left(S x_{i}, T x_{i}\right)=0, \quad \lim _{n} \sum_{j=0}^{n} \alpha_{j} \prod_{i=j+1}^{n}\left(1-\alpha_{i}+a \alpha_{i}\right) \varepsilon_{j}=0 .
$$

This completes the proof.

The following result may be derived from Theorem 3.5.

Corollary 3.6. In Theorem 3.5 if (JO) is replaced by (R), then (V) and (VI) hold with $a=k$ and $L=k /(1-k)$.

We remark that Theorems 3.4 and 3.5 extend and unify several stability results of Harder and Hicks [7, 8], Rhoades [24, 25], and Osilike [18]. In particular, Corollary 3.6 with $Y=X$ and $S=i d$ extends several results from $[8,24,25]$.

\section{Acknowledgments}

The authors thank the referees and Professor Lokenath Debnath for their appreciation, suggestions, and inspiration. Further, the authors owe to one of the referees especially for suggesting the alternative proof of Theorem 3.1.

\section{References}

[1] V. Berinde, Iterative Approximation of Fixed Points, Editura Efemeride, Baia Mare, 2002.

[2] Lj. B. Ćirić, A generalization of Banach's contraction principle, Proc. Amer. Math. Soc. 45 (1974), 267-273.

[3] L. Collatz, Functional Analysis and Numerical Mathematics, Academic Press, New York, 1966.

[4] S. Czerwik, K. Dlutek, and S. L. Singh, Round-off stability of iteration procedures for set-valued operators in b-metric spaces, J. Natur. Phys. Sci. 15 (2001), no. 1-2, 1-8.

[5] K. M. Das and K. V. Naik, Common fixed-point theorems for commuting maps on a metric space, Proc. Amer. Math. Soc. 77 (1979), no. 3, 369-373.

[6] A. M. Harder, Fixed point theory and stability results for fixed point iteration procedures, Ph.D. thesis, University of Missouri-Rolla, Missouri, 1987.

[7] A. M. Harder and T. L. Hicks, A stable iteration procedure for nonexpansive mappings, Math. Japon. 33 (1988), no. 5, 687-692.

[8] Stability results for fixed point iteration procedures, Math. Japon. 33 (1988), no. 5, 693706.

[9] V. I. Istrăţescu, Fixed Point Theory. An Introduction, Mathematics and Its Applications, vol. 7, D. Reidel, Dordrecht, 1981.

[10] J. R. Jachymski, Common fixed point theorems for some families of maps, Indian J. Pure Appl. Math. 25 (1994), no. 9, 925-937.

[11] An extension of A. Ostrowski's theorem on the round-off stability of iterations, Aequationes Math. 53 (1997), no. 3, 242-253.

[12] G. Jungck, Commuting mappings and fixed points, Amer. Math. Monthly 83 (1976), no. 4, 261263.

[13] Common fixed points for commuting and compatible maps on compacta, Proc. Amer. Math. Soc. 103 (1988), no. 3, 977-983. 
[14] W. R. Mann, Mean value methods in iteration, Proc. Amer. Math. Soc. 4 (1953), 506-510.

[15] J. Matkowski and S. L. Singh, Round-off stability of functional iterations on product spaces, Indian J. Math. 39 (1997), no. 3, 275-286.

[16] S. N. Mishra and S. L. Singh, Fixed point theorems in a locally convex space, Quaestiones Math. 19 (1996), no. 3-4, 505-515.

[17] J. M. Ortega and W. C. Rheinboldt, Iterative Solution of Nonlinear Equations in Several Variables, Academic Press, New York, 1970.

[18] M. O. Osilike, Stability results for fixed point iteration procedures, J. Nigerian Math. Soc. 14/15 (1995/96), 17-29.

[19] - A stable iteration procedure for quasi-contractive maps, Indian J. Pure Appl. Math. 27 (1996), no. 1, 25-34.

[20] A. M. Ostrowski, The round-off stability of iterations, Z. Angew. Math. Mech. 47 (1967), no. 1, 77-81.

[21] S. Park and J. S. Bae, Extensions of a fixed point theorem of Meir and Keeler, Ark. Mat. 19 (1981), no. 2, 223-228.

[22] S. Ranganathan, A fixed point theorem for commuting mappings, Math. Sem. Notes Kobe Univ. 6 (1978), no. 2, 351-357.

[23] B. E. Rhoades, A comparison of various definitions of contractive mappings, Trans. Amer. Math. Soc. 226 (1977), 257-290.

[24] - Fixed point theorems and stability results for fixed point iteration procedures, Indian J. Pure Appl. Math. 21 (1990), no. 1, 1-9.

[25] _ Fixed point theorems and stability results for fixed point iteration procedures. II, Indian J. Pure Appl. Math. 24 (1993), no. 11, 691-703.

[26] B. E. Rhoades and L. Saliga, Some fixed point iteration procedures. II, Nonlinear Anal. Forum 6 (2001), no. 1, 193-217.

[27] I. A. Rus, A. Petruşel, and G. Petruşel, Fixed Point Theory: 1950-2000. Romanian Contributions, House of the Book of Science, Cluj-Napoca, 2002.

[28] S. L. Singh, Application of a common fixed point theorem, Math. Sem. Notes Kobe Univ. 6 (1978), no. $1,37-40$.

[29] Coincidence theorems, fixed point theorems and convergence of the sequences of coincidence values, Punjab Univ. J. Math. (Lahore) 19 (1986), 83-97.

[30] A new approach in numerical praxis, Progr. Math. (Varanasi) 32 (1998), no. 2, 75-89.

[31] B. M. L. Tivari and S. L. Singh, A note on recent generalizations of Jungck contraction principle, J. Uttar Pradesh Gov. Colleges Acad. Soc. 3 (1986), no. 1, 13-18.

[32] M. Urabe, Convergence of numerical iteration in solution of equations, J. Sci. Hiroshima Univ. Ser. A. 19 (1956), 479-489.

S. L. Singh: Vedic Mathematics Research Institute, \#21 Govind Nagar, Rishikesh 249 201, India

E-mail address: vedicmri@sancharnet.in

Charu Bhatnagar: Gurukula Kangri University, Hardwar 249 404, India

E-mail address: sh42@rediffmail.com

S. N. Mishra: Department of Mathematics, University of Transkei, Umtata 5117, South Africa

E-mail address: mishra@getafix.utr.ac.za 


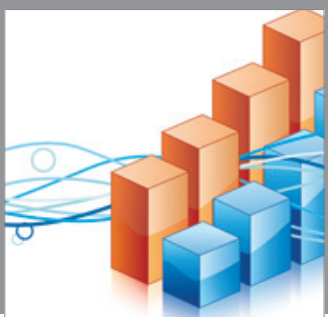

Advances in

Operations Research

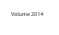

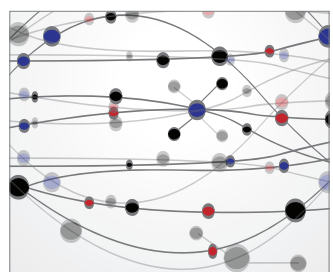

\section{The Scientific} World Journal
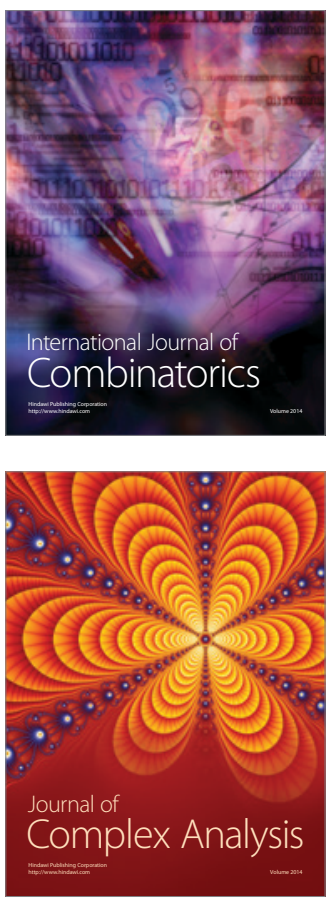

International Journal of

Mathematics and

Mathematical

Sciences
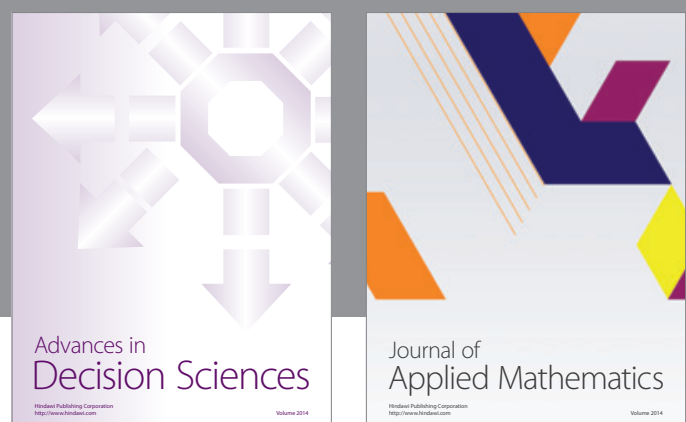

Journal of

Applied Mathematics
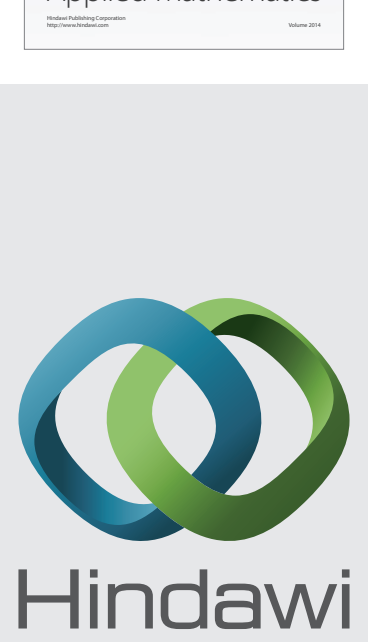

Submit your manuscripts at http://www.hindawi.com
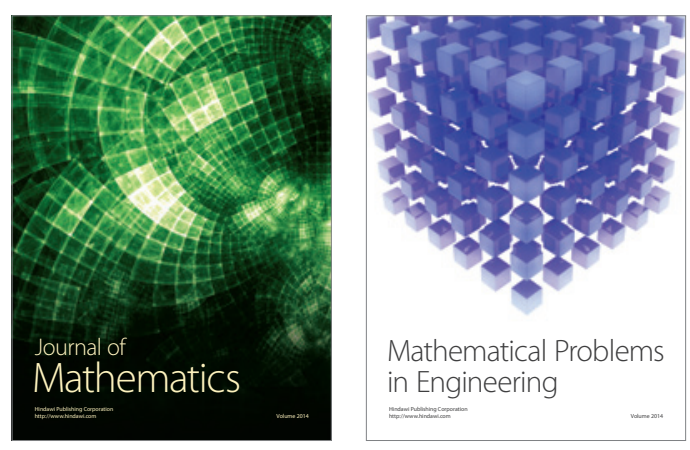

Mathematical Problems in Engineering
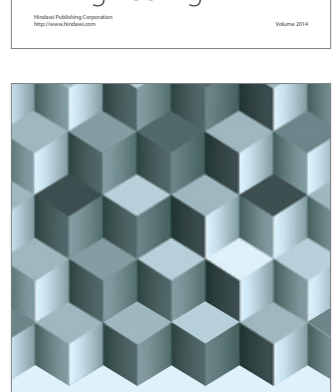

Journal of

Function Spaces
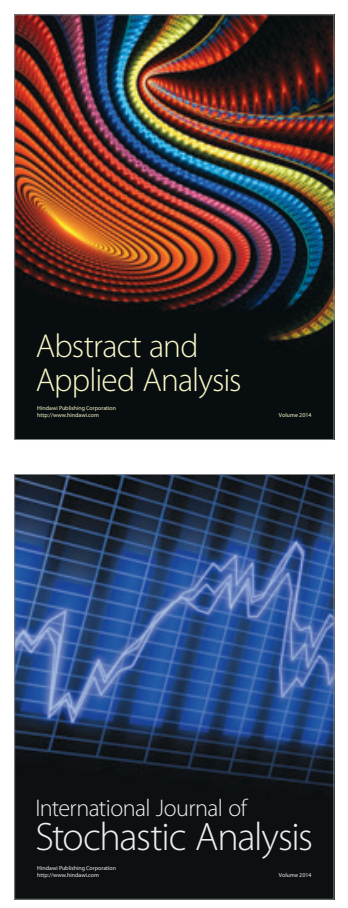

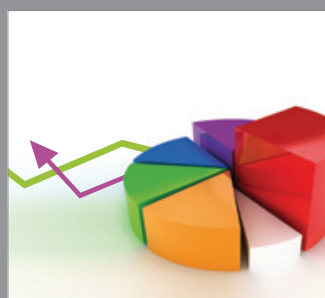

ournal of

Probability and Statistics

Promensencen
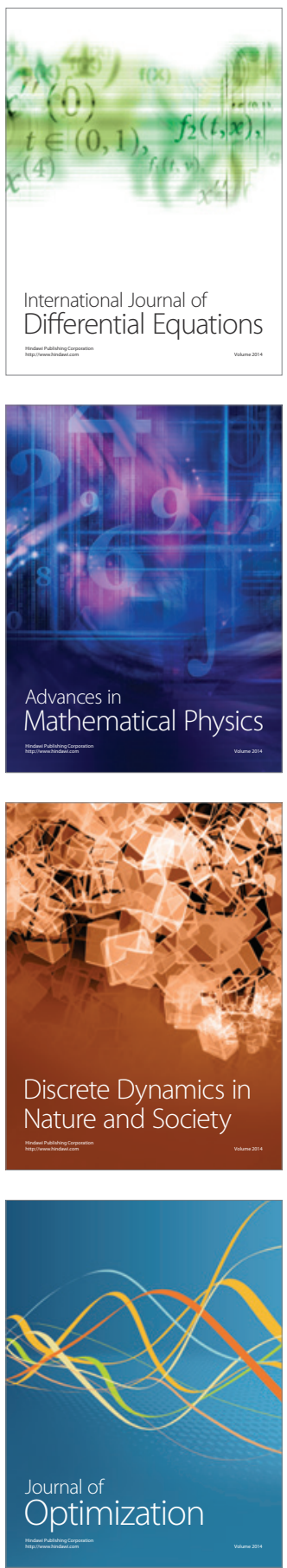